\title{
Random Access for Variable Rate Links
}

\author{
Jasvinder Singh, Chandrasekharan Raman, Roy Yates, Narayan Mandayam \\ Wireless Information Network Laboratory (WINLAB) \\ Rutgers, The State University of New Jersey \\ Technology Center of New Jersey \\ 671 Route 1 South, North Brunswick, NJ, 08902-3390 \\ \{jasingh, chandru, ryates, narayan\}@winlab.rutgers.edu
}

\begin{abstract}
We consider a model where the interfering links employ on-off modulation in each transmission slot. In the on (active) state, a link obtains a data rate determined by the interference from other active links in the network. Based on this model, we compare the throughput regions of centralized scheduling and a probabilistic random access scheme, wherein in each slot, a link is active with a fixed probability chosen independent of other interfering links. We observe that for the case of two interfering links, the probabilistic scheme does not suffer any loss in the rate region relative to the centralized scheme if the interference between the links is sufficiently low. For more than two interfering links, the characterization of throughput rate region for the probabilistic scheme becomes intractable and similar observations are not easily forthcoming. However, we give a distributed algorithm where each link independently updates its transmission probability based on its measured throughput to achieve any desired feasible rate vector in the throughput region of the probabilistic scheme and prove its convergence.
\end{abstract}

\section{INTRODUCTION}

Multiple access schemes has been a hot topic of research for more than four decades. Recently, advances in radio technology and spectrum policies have driven research to build interference aware systems like "cognitive radios" [1]. In our earlier work [2], we studied the role of "spectrum servers" as a centralized scheduler in devising fair and efficient schedule for interfering links that are capable of varying their rates of transmission. In [3], we studied the role of the spectrum server to schedule end-to-end flows in a network of interfering links. The above mentioned schemes involved centralized scheduling that requires the scheduler to know complete global information about the links. The information could be all interference gains between each pair of links in the network. More often, the availability of such global information requires a lot of overhead processing by the central entity. Hence, perfect centralized scheduling schemes act as a benchmark for imperfect scheduling schemes [4] and decentralized or distributed multiple access schemes.

Distributed random access schemes, e.g., ALOHA have been widely used in practical multiple access systems. The CSMA/CA schemes used in the IEEE 802.11 networks are very popular, thanks to the ease of implementation and decentralized control of these random access techniques. Of late, a lot of research effort has been directed towards analyzing the performance of these random access schemes. Stability properties of random access schemes have been studied in [5], [6]. In [7], [8], the authors propose distributed approaches for fair random access. The throughput characteristics of random access schemes have been studied in in [9], [10]. A recent work [11] characterizes the Pareto boundary of the network throughput region as the family of solutions optimizing a weighted proportional fairness objective, parametrized by weights chosen by the links. The authors also propose a distributed random access scheme to achieve a desired point within the Pareto optimal boundary.

In this work, we consider a model in which links turn on and off in each slot. The rate obtained in a link depends on the interference from other active links. We characterize and compare the achievable throughput region of a centralized scheduling scheme with a probabilistic random access scheme. In the centralized scheduling scheme, the scheduler provides the fraction of time a set of links are on, in order to maximize an objective function. In the probabilistic random access scheme, each link turns on or off with a fixed probability chosen independent of other links in each slot. Section III defines the throughput region of both schemes. A natural question to ask is whether the set of rates that can be achieved in both cases are the same. In section IV we attempt to characterize the throughput region of both schemes and identify conditions under which the throughput regions are the same. We derive analytic expressions for the rate region of a network with two links and provide an intuitive geometric explanation. In section $\mathrm{V}$ we then propose a distributed algorithm in which each link updates its probability of transmission based on its current rate. This memoryless policy allows to achieve any feasible point in the rate region. We prove convergence results for the distributed algorithm.

We use boldface lowercase characters for vectors and boldface uppercase for matrices. If $\mathbf{a}$ is a vector, $\mathbf{a}^{T}$ denotes its transpose and $\mathbf{a}^{T} \mathbf{b}=\sum_{i} a_{i} b_{i}$ represents the inner product of the vectors $\mathbf{a}$ and $\mathbf{b}$. The vector of all zeros and all ones are represented by $\mathbf{0}$ and $\mathbf{1}$ respectively. Inequalities between vectors are component-wise inequalities.

\section{System Model}

Consider a wireless network with $N$ nodes forming $L$ logical links sharing a common spectrum. The network can be represented as a directed graph $\mathcal{G}(\mathcal{V}, \mathcal{E})$, where the nodes in the network are represented by the set of vertices $\mathcal{V}$ of the graph and the links are represented by a set of directed edges $\mathcal{E}$. Therefore the cardinalities $|\mathcal{V}|=N$ and $|\mathcal{E}|=L$. A 


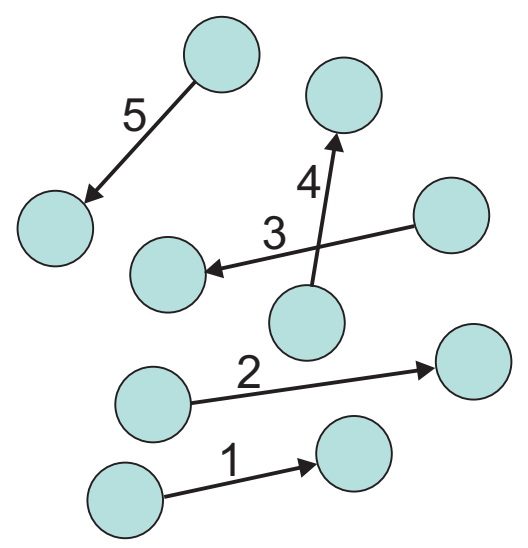

Fig. 1. Graph of network showing the nodes and directed links

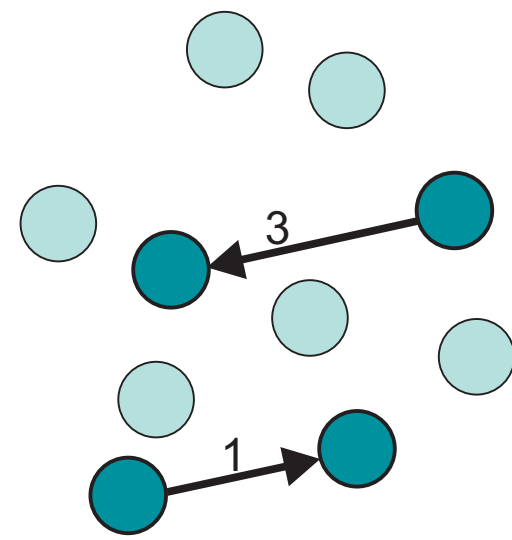

Fig. 2. Graph of network showing transmission mode corresponding to $\left(\begin{array}{llll}1 & 0 & 1 & 0\end{array}\right)$

directed edge from a node $m$ to node $n$ implies that $m$ wishes to communicate data to node $n$.

Define the set of transmission modes $\mathcal{T}=\{0,1, \ldots, M-$ $1\}$, where $M=2^{L}$ denotes the number of possible transmission modes. Then the mode activity vector $t_{j}$ of mode $j$ is a binary vector, indicating the on-off activity of the links. If $t_{j}=\left(t_{1 j}, t_{2 j}, \ldots, t_{L j}\right)$ is a mode activity vector, then

$$
t_{l j}= \begin{cases}1, & \text { link } l \text { is active under transmission mode } j, \\ 0, & \text { otherwise }\end{cases}
$$

Figure 1 shows a representative network and Figure 2 shows particular transmission mode for the set of links. Note that there are $M$ possible transmission modes including the mode in which all links are off. Let $\mathbf{T}=\left[t_{l j}\right]$ be the transmission mode matrix. Similarly, we can construct the $L \times M$ rate matrix $\mathbf{C}_{L}=\left[c_{l j}\right]$, where $c_{l j}$ is the rate obtained by link $l$ in mode $j$. By construction, $t_{l j}=0 \Rightarrow c_{l j}=0$. We impose an additional constraint on the entries of $\mathbf{C}_{L}$ : any additional interference reduces the rate of an active link. In other words, if $\mathcal{L}_{j}$ is the set of active links in mode $j$ and $\mathcal{L}_{j^{\prime}} \subset \mathcal{L}_{j}$ is the set of active links in mode $j^{\prime} \neq j$, then $c_{l j}<c_{l j^{\prime}}$ for every $l \in \mathcal{L}_{j^{\prime}} \cap \mathcal{L}_{j}$.

Many systems with interfering links can be modeled using the $\mathbf{C}_{L}$ described above, e.g., [2], [12]. The following two examples show the rate matrices for a network with two and three links respectively. For simplicity, in Sections III and IV, we will assume that each link gets a normalized rate of 1 unit, when it transmits in isolation. However, this assumption is not necessary in Section V.

$$
\begin{aligned}
\text { Example 1: } \mathbf{C}_{2}= & {\left[\begin{array}{llll}
0 & 1 & 0 & \alpha \\
0 & 0 & 1 & \beta
\end{array}\right], } \\
\text { Example 2: } \mathbf{C}_{3}= & {\left[\begin{array}{llllllll}
0 & 1 & 0 & a & 0 & c & 0 & g \\
0 & 0 & 1 & b & 0 & 0 & e & h \\
0 & 0 & 0 & 0 & 1 & d & f & i
\end{array}\right] . }
\end{aligned}
$$

The conditions for $\mathbf{C}_{2}$ are

$$
\alpha, \beta<1
$$

and the conditions for $\mathbf{C}_{3}$

$$
\begin{aligned}
a, b, c, d, e, f & <1, \\
g & <a, c, \\
h & <b, e, \\
i & <d, f .
\end{aligned}
$$

\section{RATE REGIONS}

We define the rate region as the set of rate vectors that can be achieved by a multiple access scheme. In this paper, we compare the rate regions of a centralized scheduling scheme with a probabilistic random access scheme.

\section{A. Centralized scheduling}

In this scheme, a schedule is the specified by fractions of time each transmission mode is active. A centralized scheduler can be used to compute the the optimum time fractions of activity, to maximize a certain utility function [2]. Let $x_{j}$ be the fraction of time that transmission mode $j$ is active and $r_{l}$ be the average data rate of link $l$. The average data rate in link $l$ is the time average of the data rates of all the transmission modes that include link $l$. Thus,

$$
r_{l}=\sum_{j} c_{l j} x_{j},
$$

or in vector form,

$$
\mathbf{r}=\mathbf{C}_{L} \mathbf{x}
$$

Thus the rate region for the centralized scheduling scheme is given by

$$
\mathcal{R}_{L}^{S}:=\left\{\left(r_{1}, \ldots, r_{L}\right): \mathbf{r}=\mathbf{C}_{L} \mathbf{x}, \mathbf{x} \geq 0, \mathbf{x}^{T} \mathbf{1}=1\right\} .
$$

Clearly, the region $\mathcal{R}_{L}^{S}$ is a polytope defined by its $2^{L}$ vertices which are given by the column vectors of $\mathbf{C}_{L}$.

\section{B. Random Access Scheme}

In this scheme, link $l$ transmits with a probability $p_{l}$ chosen independent of the other links in the network. The rate region for the random access scheme is given by

$$
\mathcal{R}_{L}^{P}:=\left\{\left(r_{1}, \ldots, r_{L}\right): \mathbf{r}=\mathbf{C}_{L} \mathbf{x}, \mathbf{x}=\mathbf{f}(\mathbf{p}), \mathbf{0} \leq \mathbf{p} \leq \mathbf{1}\right\}
$$


where $\mathbf{f}: \mathcal{R}^{L} \rightarrow \mathcal{R}^{2^{L}}$ is given by

$$
\mathbf{f}(\mathbf{p})=\left[\begin{array}{c}
\left(1-p_{1}\right)\left(1-p_{2}\right) \ldots\left(1-p_{L}\right) \\
p_{1}\left(1-p_{2}\right) \ldots\left(1-p_{L}\right) \\
\vdots \\
\left(1-p_{1}\right) p_{2} \ldots p_{L} \\
p_{1} \ldots p_{L}
\end{array}\right] .
$$

It is easy to see that $\mathcal{R}_{L}^{P} \subseteq \mathcal{R}_{L}^{S}$. Also, since $\mathbf{f}($.$) is a continuous$ mapping, the set $\{\mathbf{x}: \mathbf{x}=\mathbf{f}(\mathbf{p}), 0 \leq \mathbf{p} \leq 1\}$ must be a closed and continuous region and therefore $\mathcal{R}_{L}^{P}$ must also be closed and continuous. Our aim will be to characterize the Pareto boundary of $\mathcal{R}_{L}^{P}$ and find out the conditions, if any, under which $\mathcal{R}_{L}^{P} \equiv \mathcal{R}_{L}^{S}$. We first consider the following simple cases for getting some insight into the shape of the rate regions.

\section{Characterization of $\mathcal{R}_{L}^{P}$}

\section{A. $L=2$}

Using (10) and definition of $\mathbf{C}_{2}$ from Example 1, the rates on two links are

$$
\begin{aligned}
& r_{1}=p_{1}\left(1-p_{2}\right)+\alpha p_{1} p_{2}, \\
& r_{2}=\left(1-p_{1}\right) p_{2}+\beta p_{1} p_{2}
\end{aligned}
$$

The above equations can be rewritten as

$$
\begin{aligned}
& r_{1}=p_{2}\left(p_{1} \alpha+\left(1-p_{1}\right) \cdot 0\right)+\left(1-p_{2}\right)\left(p_{1} \cdot 1+\left(1-p_{1}\right) \cdot 0\right), \\
& r_{2}=p_{2}\left(p_{1} \beta+\left(1-p_{1}\right) \cdot 1\right)+\left(1-p_{2}\right)\left(p_{1} \cdot 0+\left(1-p_{1}\right) \cdot 0\right) .
\end{aligned}
$$

In vector form,

$$
\begin{aligned}
{\left[\begin{array}{l}
r_{1} \\
r_{2}
\end{array}\right] } & =p_{2}\left(p_{1}\left[\begin{array}{l}
\alpha \\
\beta
\end{array}\right]+\left(1-p_{1}\right)\left[\begin{array}{l}
0 \\
1
\end{array}\right]\right) \\
& +\left(1-p_{2}\right)\left(p_{1}\left[\begin{array}{l}
1 \\
0
\end{array}\right]+\left(1-p_{1}\right)\left[\begin{array}{l}
0 \\
0
\end{array}\right]\right)
\end{aligned}
$$

The above representation of the rate vector, as a nested convex combination of the polytope vertices, is useful in visualizing the rate region $\mathcal{R}_{2}^{P}$. We now consider two different cases.

1) $\alpha+\beta \geq 1$ : Figure 3 shows $\mathcal{R}_{2}^{S}$. Any point in the quadrilateral $O A B C$ can be achieved using centralized scheduling. Notice that the vertices of the polytope $O A B C$ are the columns of $\mathbf{C}_{2}$. For a given probability vector $\mathbf{p}=\left[\begin{array}{ll}p_{1} & p_{2}\end{array}\right]^{T}$, the rate vector $\mathbf{r}$ given by (16) is shown as point $F$ in Figure 3. As $p_{1}$ varies between 0 and 1 , points $D$ and $E$ completely trace the line segments $A B$ and $O C$ respectively. As $p_{2}$ varies between 0 and 1 , the point $F$ traverses the line segment $E D$ completely. Hence, it can be seen that by varying $\mathbf{p}$, the achieved rate region $\mathcal{R}_{L}^{P}$ is the same as $\mathcal{R}_{L}^{S}$.

Analytically, we can write (derivation given in the Appendix VI-A)

$$
\mathcal{R}_{L}^{P}=\left\{\begin{array}{c}
\left(r_{1}, r_{2}\right): \\
0 \leq r_{1} \leq \alpha \Rightarrow 0 \leq r_{2} \leq \frac{\alpha-(1-\beta) r_{1}}{\alpha} \\
\alpha \leq r_{1} \leq 1 \quad \Rightarrow \quad 0 \leq r_{2} \leq \frac{\beta\left(1-r_{1}\right)}{1-\alpha}
\end{array}\right\}
$$

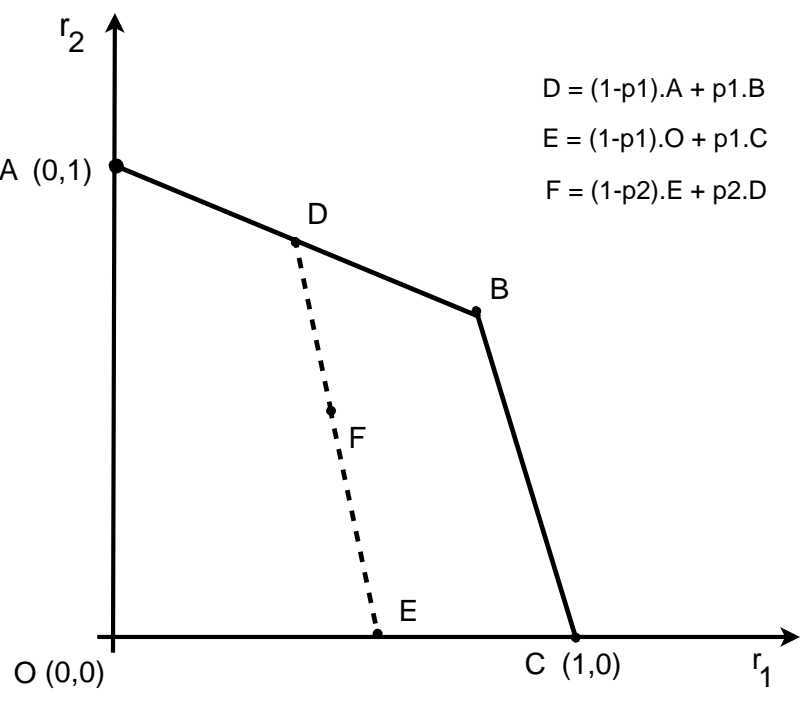

Fig. 3. $\quad \mathcal{R}_{L}^{S}$ and $\mathcal{R}_{L}^{P}$ for the case $\alpha+\beta \geq 1$. $\mathcal{R}_{L}^{P} \equiv \mathcal{R}_{L}^{S}$ and is given by the area enclosed by $O A B C$. B represents $(\alpha, \beta)$.

2) $\alpha+\beta<1$ : In this case, $\mathcal{R}_{L}^{S}$ is given by the triangle formed by points $O, A$ and $C$ in Figure 4. As in the previous case, point $F$ in Figure 4 corresponds to the rate vector $\mathbf{r}$ achieved for a given $\mathbf{p}=\left[p_{1} p_{2}\right]^{T}$. If $p_{1}=1$, the line segment $D E$ coincides with $B C$. As $p_{1}$ varies from 1 to $0, D E$ moves from $B C$ to an intermediate position $H G$ to finally $A O$ (for $p_{1}=0$ ) tracing out the region $\mathcal{R}_{L}^{P}$ as the area enclosed by $O A H I C$. Note that the boundary $A H I C$ of the region is convex (verified from the analytical expression for $\mathcal{R}_{L}^{P}$ in the appendix) and contains two linear components $A H$ and $I C$. The presence of linear component $A H$ can be geometrically understood by observing that as $D E$ moves from $H G$ to $A O$, endpoint $D$ always lies on the linear segment $A H$. In order to intuitively understand the presence of $I C$, it helps to notice that as $p_{1}$ varies from 1 to $0, J$, the point of intersection of $D E$ and $B C$ initially moves from $B$ towards $C$, goes up to a certain point $I$, and then moves back towards $B$.

Note that we could also have expressed the rate equations as

$$
\begin{aligned}
{\left[\begin{array}{l}
r_{1} \\
r_{2}
\end{array}\right] } & =p_{1}\left(p_{2}\left[\begin{array}{l}
\alpha \\
\beta
\end{array}\right]+\left(1-p_{2}\right)\left[\begin{array}{l}
1 \\
0
\end{array}\right]\right) \\
& +\left(1-p_{1}\right)\left(p_{2}\left[\begin{array}{l}
0 \\
1
\end{array}\right]+\left(1-p_{2}\right)\left[\begin{array}{l}
0 \\
0
\end{array}\right]\right)
\end{aligned}
$$

The above equations give an alternate equivalent way of looking at the region, where now instead of lines $A B$ and $O C$, we consider lines $B C$ and $A O$.

The analytical characterization of the above region is given below (derivation given in the Appendix VI-A) 


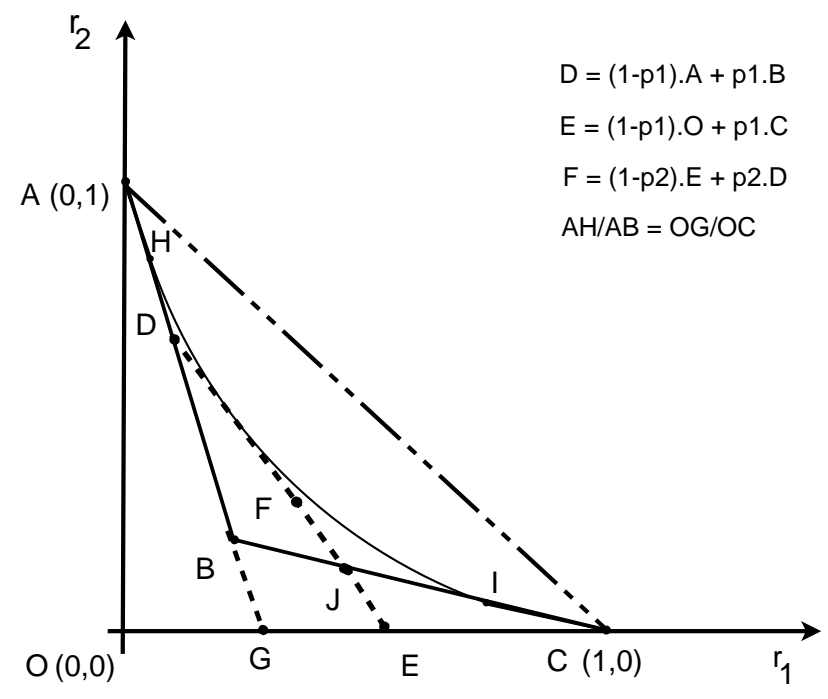

Fig. 4. $\mathcal{R}_{L}^{S}$ and $\mathcal{R}_{L}^{P}$ for the case: $\alpha+\beta<1$. $\mathcal{R}_{L}^{S}$ is given by the area enclosed by $O A C$ and $\mathcal{R}_{L}^{P}$ is given by the area enclosed by $O A H I C$. $B=(\alpha, \beta)$.

$\mathcal{R}_{L}^{P}=\left\{\begin{array}{cc}\left(r_{1}, r_{2}\right): & \\ 0 \leq r_{1} \leq \frac{\alpha^{2}}{1-\beta} \Rightarrow & 0 \leq r_{2} \leq \frac{\alpha-(1-\beta) r_{1}}{\alpha}, \\ \frac{\alpha^{2}}{1-\beta}<r_{1}<1-\beta \Rightarrow & 0 \leq r_{2} \leq \frac{\left(\sqrt{(1-\beta) r_{1}}-1\right)^{2}}{1-\alpha}, \\ 1-\beta \leq r_{1} \leq 1 \Rightarrow & 0 \leq r_{2} \leq \frac{\beta\left(1-r_{1}\right)}{1-\alpha} .\end{array}\right\}$

\section{B. $L=3$}

The analytical characterization of the rate region is cumbersome for the three dimensional case because of the number of sub-cases that need to be considered. However, the geometric intuition that we developed for the two link case can easily be extended to this case. Using the definition of $\mathbf{C}_{3}$, we can write the rate vector $\mathbf{r}(\mathbf{p})$ in the following form:

$$
\begin{aligned}
{\left[\begin{array}{l}
r_{1} \\
r_{2} \\
r_{3}
\end{array}\right] } & =p_{3}\left\{p_{2}\left(p_{1}\left[\begin{array}{l}
g \\
h \\
i
\end{array}\right]+\left(1-p_{1}\right)\left[\begin{array}{l}
0 \\
e \\
f
\end{array}\right]\right)\right. \\
& \left.+\left(1-p_{2}\right)\left(p_{1}\left[\begin{array}{l}
c \\
0 \\
d
\end{array}\right]+\left(1-p_{1}\right)\left[\begin{array}{l}
0 \\
0 \\
1
\end{array}\right]\right)\right\} \\
& +\left(1-p_{3}\right)\left\{p_{2}\left(p_{1}\left[\begin{array}{l}
a \\
b \\
0
\end{array}\right]+\left(1-p_{1}\right)\left[\begin{array}{l}
0 \\
1 \\
0
\end{array}\right]\right)\right. \\
& \left.+\left(1-p_{2}\right)\left(p_{1}\left[\begin{array}{l}
1 \\
0 \\
0
\end{array}\right]+\left(1-p_{1}\right)\left[\begin{array}{l}
0 \\
0 \\
0
\end{array}\right]\right)\right\}
\end{aligned}
$$

Figure 5 illustrates the nested convex combination structure given above, where point $N$ corresponds to the rate vector $\mathbf{r}(\mathbf{p})$. Working with our geometric intuition, we make the following claim (without proof):

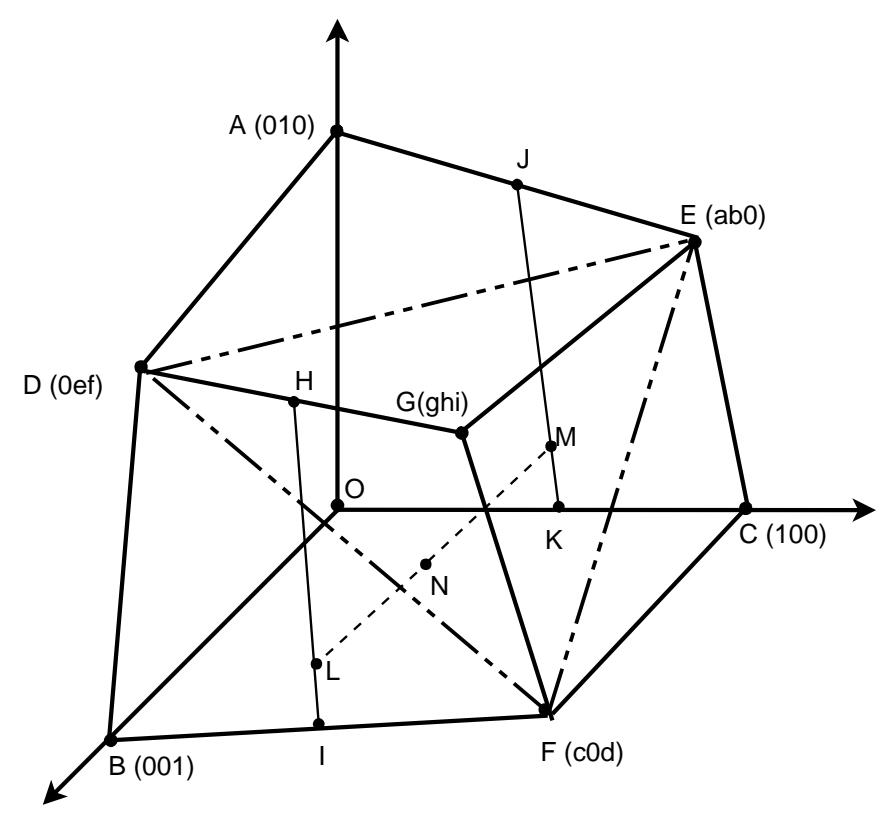

Fig. 5. Visualizing the rate region for the $L=3$ case

Claim 1: $\mathcal{R}_{L}^{P} \equiv \mathcal{R}_{L}^{S} \Leftrightarrow$ following conditions are satisfied:

- $a+b \geq 1, c+d \geq 1, e+f \geq 1$.

- Points $\{(0,0,1),(c, 0, d),(g, h, i),(0, e, f)\}$ are coplanar.

- Points $\{(1,0,0),(a, b, 0),(g, h, i),(c, 0, d)\}$ are coplanar.

- Points $\{(0,1,0),(0, e, f),(g, h, i),(a, b, 0)\}$ are coplanar.

\section{Distributed Algorithm}

In this section, we present a distributed random access algorithm to achieve a feasible point in the rate region $\mathcal{R}_{L}^{P}$ for a network with $L$ links. Each link updates its probability of transmission based on the rate it achieves in the previous slot. We start by identifying a property of the function $r_{i}(\mathbf{p})$ (Lemma 1) that is the key for proving the convergence of our distributed algorithm.

The rate $r_{i}(\mathbf{p})$ achieved by link $i$ in the random access scheme can be written as

$$
\begin{aligned}
r_{i}(\mathbf{p}) & =\sum_{j=1}^{M} c_{i j} \prod_{l=1}^{L}\left[t_{l j} p_{l}+\left(1-t_{l j}\right)\left(1-p_{l}\right)\right] \\
& =p_{i} \sum_{j: t_{i j}=1} c_{i j} \prod_{l \neq i}\left[t_{l j} p_{l}+\left(1-t_{l j}\right)\left(1-p_{l}\right)\right]
\end{aligned}
$$

Let us define

$$
g_{i}\left(\mathbf{p}_{-i}\right)=\sum_{j: t_{i j}=1} c_{i j} \prod_{l \neq i}\left[t_{l j} p_{l}+\left(1-t_{l j}\right)\left(1-p_{l}\right)\right]
$$

where

$$
\mathbf{p}_{-i}=\left[p_{1}, \ldots, p_{i-1}, p_{i+1}, \ldots, p_{L}\right]^{T}
$$

Then $r_{i}(\mathbf{p})$ can be written as

$$
r_{i}(\mathbf{p})=p_{i} g_{i}\left(\mathbf{p}_{-i}\right)
$$


Lemma 1: $g_{i}($.$) is a positive and strictly decreasing function$ of $p_{j}$ for all $j \neq i$. Therefore, $r_{i}($.$) is a strictly increasing$ function of $p_{i}$ and a strictly decreasing function of $p_{j}$ for all $j \neq i$.

Proof: See Appendix VI-B.

Now for each link $i$, consider the following iterative update of $p_{i}(n)$ based on the current rate $r_{i}(n)$ and the desired rate $r_{i}^{d}$. In practice the current rate $r_{i}(n)$ is measured by averaging the rates obtained over many slots.

$$
p_{i}(n+1)=\frac{r_{i}^{d}}{r_{i}(n)} p_{i}(n)
$$

Theorem 1: Given a feasible rate vector $\mathbf{r}^{d} \in \mathcal{R}_{L}^{P}$, if all the links perform the above iteration independently starting with $\mathbf{p}(0)=\mathbf{0}$, then their iterations converge to a fixed point $\left(\mathbf{p}^{*}, \mathbf{r}^{*}\right)$ such that $\mathbf{r}^{*}=\mathbf{r}^{d}$ and $\mathbf{p}(n) \leq \mathbf{1}$ for all $n$.

Proof: Using (26), we can rewrite (27) as

$$
p_{i}(n+1)=\frac{r_{i}^{d}}{g_{i}\left(\mathbf{p}_{-i}(n)\right)}
$$

Substituting $\mathbf{p}(0)=\mathbf{0}$ in the iteration, we get $\mathbf{p}(1)=\mathbf{r}^{d}$ and therefore $\mathbf{p}(1) \geq \mathbf{p}(0)$. Using lemma 1 with the above fact, it follows that $\mathbf{p}(2) \geq \mathbf{p}(1)$ and in general $\mathbf{p}(n+1) \geq \mathbf{p}(n)$ for all $n$. Therefore, if $\mathbf{p}(n)$ is bounded from above by $\mathbf{1}$, as $n$ increases, it must converge to a fixed point $\mathbf{p}^{*}$ and the corresponding $\mathbf{r}^{*}$ is then equal to $\mathbf{r}^{d}$.

Now we prove that if $\mathbf{r}^{d}$ is feasible, then $\mathbf{p}(n)$ remains bounded below 1. Feasibility of $\mathbf{r}^{d}$ means that there exists $\mathbf{0} \leq \mathbf{p}^{d} \leq \mathbf{1}$ such that

$$
p_{i}^{d}=\frac{r_{i}^{d}}{g_{i}\left(\mathbf{p}_{-i}^{d}(n)\right)}
$$

By definition, $\mathbf{p}^{d} \geq \mathbf{p}(0)$. Using (28) and (29), we can see that $\mathbf{p}^{d} \geq \mathbf{p}(1)$ and in general $\mathbf{p}^{d} \geq \mathbf{p}(n)$ for all $n$. Therefore $\mathbf{p}(n)$ must also remain bounded below $\mathbf{1}$.

In case the users choose an infeasible $r^{d}$, the above iteration will lead to a situation where some $p_{i}(n)$ 's exceed 1 . To fix this, we can modify the iteration to the one given below.

$$
p_{i}(n+1)=\min \left\{\frac{r_{i}^{d}}{r_{i}(n)} p_{i}(n), 1\right\}
$$

The above iteration converges to the desired rate vector $\mathbf{r}^{d}$ if $\mathbf{r}^{d}$ is feasible. In case the users start with an infeasible $\mathbf{r}^{d}$, we make some simple observations that are stated below as Lemma 2. Let $\mathbf{r}^{1}$ denote the rate vector corresponding to $\mathbf{p}=\mathbf{1}$ and $\left(\mathbf{p}^{*}, \mathbf{r}^{*}\right)$ denote the probability and rate vectors obtained when the above iteration converges for all $i$.

Lemma 2: Assume $\mathbf{r}^{d}$ is infeasible. Then for any link $i$, the following conditions hold true: (a) $r_{i}^{d}>r_{i}^{1} \Rightarrow r_{i}^{*} \geq r_{i}^{1}$, (b) $r_{i}^{d} \leq r_{i}^{1} \Rightarrow r_{i}^{*}=r_{i}^{d}$. (c) If $L=2$, then $\mathbf{r}^{d}>\mathbf{r}^{1} \Rightarrow \mathbf{r}^{*}=\mathbf{r}^{1}$.

Proof: (a) Clearly, $\mathbf{p}^{*} \leq \mathbf{1}$. If $p_{i}^{*}=1$, then $r_{i}^{*}=$ $1 . g_{i}\left(\mathbf{p}_{-i}^{*}\right) \geq 1 . g_{i}(\mathbf{1})=r_{i}^{1}$ (using Lemma 1). If $p_{i}^{*}<$ 1 , then using the convergence condition we get, $p_{i}^{*}=$ $\min \left\{p_{i}^{*} r_{i}^{d} / r_{i}^{*}, 1\right\}=p_{i}^{*} r_{i}^{d} / r_{i}^{*}$ which gives $r_{i}^{*}=r_{i}^{d}>r_{i}^{1}$. (b) We prove this by showing that for all $n$, $\min \left\{p_{i}(n) r_{i}^{d} / r_{i}(n), 1\right\}=p_{i}(n) r_{i}^{d} / r_{i}(n)$ which implies (using proof of Theorem 1) that $r_{i}^{*}=r_{i}^{d}$. To see that $p_{i}(n) r_{i}^{d} / r_{i}(n) \leq 1$ for all $n$, consider the following chain of inequalities using Lemma 1:

$$
\frac{r_{i}^{d}}{r_{i}(n)} p_{i}(n)=\frac{r_{i}^{d}}{g_{i}\left(\mathbf{p}_{-i}(n)\right)} \leq \frac{r_{i}^{d}}{g_{i}(\mathbf{1})} \leq \frac{r_{i}^{1}}{g_{i}(\mathbf{1})}=1
$$

(c) We want to show that if $\mathbf{r}^{d}$ is infeasible and $\mathbf{r}^{d}>\mathbf{r}^{1}$, then $p_{1}^{*}=p_{2}^{*}=1$. We prove this by contradiction. It is not possible that both $p_{1}^{*}<1$ and $p_{2}^{*}<1$ because this would then imply that $\mathbf{r}^{d}$ was feasible. Without loss of generality assume that $p_{1}^{*}<1$ and $p_{2}^{*}=1$. Then we can write $r_{1}^{d}=r_{1}\left(p_{1}^{*}, 1\right) \leq$ $r_{1}(1,1)=r_{1}^{1}<r_{1}^{d}$ (using Lemma 1 ) which is a contradiction.

\section{CONCLUSIONS}

In this paper, we compared the achievable throughput region of a probabilistic transmission scheme with that of centralized scheduling. We also presented a distributed algorithm to achieve any feasible rate vector in the throughput region of the probabilistic transmission scheme and proved its convergence.

\section{APPENDIX}

A. Derivation of the pareto-boundary of the rate region for $L=2$ case

Let $\gamma_{1}=1-\alpha$ and $\gamma_{2}=1-\beta$. Then we can write (12) and (13) as,

$$
\begin{aligned}
& r_{1}=p_{1}\left(1-\gamma_{1} p_{2}\right), \\
& r_{2}=p_{2}\left(1-\gamma_{2} p_{1}\right) .
\end{aligned}
$$

The Pareto boundary of the rate region is obtained by maximizing $r_{2}$ (or $r_{1}$ ) for each value of $r_{1}$ (or $r_{2}$ ). The constraints are $0 \leq p_{1}, p_{2} \leq 1$. If we substitute $p_{1}=r_{1} /\left(1-\gamma_{1} p_{2}\right)$ in the expression for $r_{2}$, then constraints $0 \leq p_{1} \leq 1$ imply that

$$
0 \leq p_{2} \leq \min \left\{\frac{1-r_{1}}{\gamma_{1}}, 1\right\} \text {. }
$$

Now, for a given $r_{1} \in[0,1]$, we can find $r_{2}$ that lies on the Pareto boundary by solving the following optimization problem:

$$
\begin{aligned}
& \qquad r_{2}=\max _{p_{2}}\left(p_{2}-\frac{\gamma_{2} p_{2} r_{1}}{1-\gamma_{1} p_{2}}\right) \\
& \text { subject to } \quad 0 \leq p_{2} \leq \min \left\{\frac{1-r_{1}}{\gamma_{1}}, 1\right\} .
\end{aligned}
$$

Since $r_{2}=0$ at $p_{2}=0$, the maximum occurs either at the boundary point $p_{2}=\min \left\{\left(1-r_{1}\right) / \gamma_{1}, 1\right\}$ or at a point where the derivative of the above function w.r.t. $p_{2}$ is zero. Setting the derivative $r_{2}^{\prime}\left(p_{2}\right)=0$ gives $p_{2}=\left(1 \pm \sqrt{\gamma_{2} r_{1}}\right) / \gamma_{1}$. One of these values is greater than 1 and can be discarded. For the other value of $p_{2}$ to be valid, we need

$$
0 \leq \frac{1-\sqrt{\gamma_{2} r_{1}}}{\gamma_{1}} \leq \min \left\{\frac{1-r_{1}}{\gamma_{1}}, 1\right\},
$$


which is satisfied only if $\left(1-\gamma_{1}\right)^{2} / \gamma_{2} \leq r_{1} \leq \gamma_{2}$. Rewriting in terms of $\alpha$ and $\beta$,

$$
\frac{\alpha^{2}}{1-\beta} \leq r_{1} \leq 1-\beta
$$

We now consider the following two cases:

1) $\alpha+\beta \geq 1$ : In this case, the maximum value of $r_{2}$ always occurs at the boundary point $p_{2}=\min \left\{\left(1-r_{1}\right) /(1-\right.$ $\alpha), 1\}$. The optimal value of $p_{2}$ that maximizes $r_{2}$ is either $\left(1-r_{1}\right) /(1-\alpha)$ or 1 depending on whether $r_{1} \geq \alpha$ or not. Substituting the maximizing value of $p_{2}$ in (34), we obtain the rate region given by (17).

2) $\alpha+\beta<1$ : In this case, we have to consider separately the following ranges of $r_{1}$ :

$$
\begin{aligned}
0 & \leq r_{1} \leq \frac{\alpha^{2}}{1-\beta}, \\
\frac{\alpha^{2}}{1-\beta} & <r_{1}<1-\beta, \\
1-\beta & \leq r_{1} \leq 1 .
\end{aligned}
$$

The optimal values of $p_{2}$ corresponding to the above three ranges of $r_{1}$ are $p_{2}=\left[1, \frac{1-\sqrt{(1-\beta) r_{1}}}{1-\alpha}, \frac{1-r_{1}}{1-\alpha}\right]$. Substituting in (34), we obtain the rate region given by (19).

\section{B. Proof of lemma 1}

Positivity of $g_{i}($.$) is evident from its definition. We must$ now show that $g_{i}($.$) is a strictly decreasing function of p_{k}$ for all $k \neq i$. Computing the partial derivative of $g_{i}$ w.r.t. $p_{k}$ we get

$$
\frac{\partial g_{i}}{\partial p_{k}}=\sum_{j: t_{i j}=1} c_{i j}\left(2 t_{k j}-1\right) \prod_{l \neq i, l \neq k}\left[t_{l j} p_{l}+\left(1-t_{l j}\right)\left(1-p_{l}\right)\right]
$$

In the above expression, the index $j$ counts all the transmission modes in which $t_{i j}=1$. Lets denote this set by $\mathcal{T}_{i=1}$. The set $\mathcal{T}_{i=1}$ can be partitioned into two disjoint sets $\mathcal{T}_{i=1, k=1}$ and $\mathcal{T}_{i=1, k=0}$ depending on whether the link $k$ is active or not. Then for each $j \in \mathcal{T}_{i=1, k=1}$, there exists a unique $j^{\prime} \in \mathcal{T}_{i=1, k=0}$ such that $t_{l j}=t_{l j^{\prime}}$ for all $l \neq k$. Now noting that $j \in \mathcal{T}_{i=1, k=1}$ implies by definition that $\left(2 t_{k j}-1\right)=1$ and similarly $j^{\prime} \in \mathcal{T}_{i=1, k=0}$ implies that $\left(2 t_{k j^{\prime}}-1\right)=-1$, we can write [ 37] as

$$
\frac{\partial g_{i}}{\partial p_{k}}=\sum_{j \in \mathcal{T}_{i=1, k=1}}\left(c_{i j}-c_{i j^{\prime}}\right) \prod_{l \neq i l \neq k}\left[t_{l j} p_{l}+\left(1-t_{l j}\right)\left(1-p_{l}\right)\right]
$$

Using the properties of $C_{L}$, we know that $c_{i j}<c_{i j^{\prime}}$ for all $j \in \mathcal{T}_{i=1, k=1}$. Therefore the above expression is negative and the result follows.

\section{ACKNOWLEDGEMENT}

This work is supported in part by the NSF under grant number NeTS-0434854 and by the Defense Spectrum Office (DSO) of the Defense Information Systems Agency.

\section{REFERENCES}

[1] S. Haykin, "Cognitive radio: brain-empowered wireless communications," IEEE Journal on Selected Areas in Communications, vol. 23, pp. 201-220, Feb 2005.

[2] C. Raman, R. Yates, and N. Mandayam, "Scheduling variable rate links via a spectrum server," in Proc. IEEE DySPAN, 2005. Baltimore, MD.

[3] C. Raman, R. Yates, and N. Mandayam, "Cross layer scheduling of endto-end flows using a spectrum server," in Proc. CISS, 2006. Princeton, NJ.

[4] X. Lin and N. B. Shroff, "The impact of imperfect scheduling on crosslayer rate control in multihop wireless networks," in IEEE Infocom, 2005

[5] B. Hajek and T. V. Loon, "Decentralized dynamic control of a multiaccess broadcast channel," IEEE Trans. Auto. Control, vol. 27, pp. 559569, Jun 1982.

[6] S. Ghez, S. Verdu, and S. C. Schwartz, "Stability properties of slotted aloha with multipacket reception capability," IEEE Trans. Auto. Control, vol. 33, pp. 640-649, Jul 1988.

[7] K. Kar, S. Sarkar, and L. Tassiulas, "Achieving proportional fairness using local information in aloha networks," IEEE Trans. Auto. Control, vol. 49, pp. 1858-1862, Oct 2004.

[8] X. Wang and K. Kar, "Distributed approaches for proportional and maxmin fairness in random access ad hoc networks," in Proc. CISS, 2006. Princeton, NJ.

[9] J. Massey and P. Mathys, "The collision channel without feedback," IEEE Trans. Info. Theory, vol. 31, pp. 192-204, Mar 1985.

[10] L. Kleinrock and F. Tobagi, "Packet switching in radio channels: Part i: Carrier sense multiple access and their throughput delay characteristics," IEEE Trans. Communications, vol. 23, pp. 1400-1412, Dec 1975.

[11] P. Gupta and A. Stolyar, "Optimal throughput allocation in general random-access networks," in Proc. CISS, 2006. Princeton, NJ.

[12] B. Radunovic and J. Y. L. Boudec, "Rate performance objectives of multihop wireless networks," IEEE Trans. Mobile Computing, vol. 3, pp. 334-349, Oct.-Dec 2004. 